\title{
Integration of an Agent-based Strategic Planner in an Enterprise Service Bus Ecosystem
}

\author{
Adriano Ferreira ${ }^{1}$, Arnaldo Pereira ${ }^{1}$, Nelson Rodrigues ${ }^{1,2}$, José Barbosa $^{1}$, Paulo Leitão ${ }^{1,2}$ \\ ${ }^{1}$ Polytechnic Institute of Bragança, Campus Sta Apolónia, Apartado 1134, 5301-857 Bragança, Portugal \\ \{a.ferreira, arnaldo, nrodrigues, jbarbosa, pleitao\}@ipb.pt \\ ${ }^{2}$ Artificial Intelligence and Computer Science Laboratory, Rua Campo Alegre 102, 4169-007 Porto, Portugal
}

\begin{abstract}
The continuous change in the manufacturing world is demanding more flexible, responsive and accurate planning tools, which are able to assist the decision-makers to take tactical and strategic decisions on short notice with a high level of confidence. For this purpose, these tools should dynamically explore different operative scenarios in the planning procedure and produce information about key performance indicators. This paper describes the development of an agentbased strategic planner, combining the flexibility of multi-agent systems principles with the optimization capability of a Mixed Integral Programming technique. The tool is integrated in an ecosystem of heterogeneous decision-making systems through an Enterprise Service Bus that also provides access to legacy data.
\end{abstract}

\section{INTRODUCTION}

In the last years, the increased worldwide economic competition made reliability, responsiveness, resilience and agility critical issues. To avoid being surprised when unexpected events occur, companies need to use responsive optimization tools that will provide reliable results to accommodate the constant occurrence of such events, e.g., nonconformities, resources breakdown, demand fluctuation, late requests for change and immature technology. This problem is even more challenging for the production planning and control in the ramp-up of complex and highly customized products, particularly in small lot sizes, as in aircraft and shipbuilding industries. The daily unexpected events create significant risks in the first products of a series. Additionally, as these products are made in short series, learning from these events becomes difficult and slow. As conclusion, the challenge is to achieve optimized solutions in very short time, incorporating in a short notice the learning from these unexpected events.

The ARUM (Adaptive Production Management) project [1] is developing mitigation strategies to respond faster and more properly to unexpected events in the ramp-up production of complex and highly customized products, namely in situations of peak of demand, late requests and immature technology. The architectural principles are based on an ecosystem of heterogeneous agent-based real-time decision-support tools that are able to early detect warnings and to accelerate the learning process. These tools are integrated by means of an ESB (Enterprise Service Bus) platform.

In this context, one important tool is the strategic planner that addresses the optimization of the resource allocation considering specific constraints (e.g., dependencies and availabilities), subject to different criteria (e.g., minimizing the labour or inventory costs) and considering a long time horizon. Particularly, the tool should be able to produce and analyse alternative planning solutions for situations where unexpected events may provoke strong impact on the existing plans.

The traditional solution for the production planning is to consider a solver as the mean to reach possible planning solutions for given constraints. The solver runs the optimization method for the specific problem formulation using the current context [2]. The optimization methods range from linear programming to meta-heuristics, such as local search methods and evolutionary algorithms. Several solvers are currently available, namely IBM ILOG CPLEX Optimizer, Xpress Optimization Suite, MOSEK, Gurobi Optimizer and KNITRO (see [3] for a comparative analysis). In spite of their optimization levels, these tools lack the responsiveness to achieve solutions in short term, which is crucial in industrial environments that are usually subject to condition changes.

An alternative is to consider the MAS (Multi-Agent Systems) paradigm to implement the optimization algorithm in a distributed manner. This approach allows to achieve flexibility, robustness and responsiveness in achieving planning solutions but the achieved optimization level is degraded. Examples of the application of MAS principles in production planning are [4-6].

In this work, a hybrid approach is considered for the production planning problem, combining the best of the two worlds: a solver to ensure maturity, stability and optimization and a MAS to provide flexibility and responsiveness to address the complex ramp-up problem. Some works considering these ideas are already reported in the literature, namely [7]. The difference to these works are mainly centred on using MAS to provide a what-if game simulation to explore different DoF (Degrees of Freedom), e.g., capacity expansion possibilities to accommodate the demand fluctuation.

This paper describes the development of such MAS strategic planning solution and its posterior integration in an ESB platform, allowing to achieve interoperability and plugability using the service-orientation principles. These platforms also allow the transparent access to data from legacy systems, such as ERP (Enterprise Resource Planning) and MES (Manufacturing Execution Systems).

The application of the proposed strategic planner is illustrated with a use case related to the increase of the production demand in $10 \%$ a year for a specific product. The 